links TNFR1 to FADD. In addition to the apoptosis-inducing activity, TNFR1, and also Fas, can activate the transcription factor NF- $\kappa \mathrm{B}$, which leads to the expression of anti-apoptotic molecules, as well as inflammatory cytokines. In accordance with the apoptosis-inducing activity of FasL, a mutation in Fas or FasL in mice or humans causes hyperplasia in lymphocytes, which sometimes leads to neoplastic transformation ${ }^{12}$. On the other hand, mice deficient in TNF do not show hyperplasia, but are resistant to endotoxin-induced lethal shock, indicating that the main in vivo function of TNF is to regulate the immune reactions, rather than to kill cells.

FasL and TNF are mainly expressed in activated $\mathrm{T}$ cells and/or macrophages. TRAIL is expressed in many human tissues, although not in the liver and brain $^{5}$, which indicates that TRAIL does not have a cytotoxic effect on normal cells. In fact, many normal primary cells such as epithelial cells, fibroblasts, and skeletal muscle cells are resistant to TRAIL-induced apoptosis ${ }^{7}$. Hepatocytes from mouse and monkey are resistant to TRAIL (ref. 9), which explains why the lack of liver damage when TRAIL was injected into these species. However, Jo et al. have shown that human primary hepatocytes are very sensitive to TRAIL-induced apoptosis ${ }^{9}$.

TRAIL has two death-domain-containing receptors (DR4 and DR5). In addition, there are two 'decoy' receptors for TRAIL, DcR1 and DcR2, which lack the cytoplasmic region and have a truncated death domain, respectively ${ }^{11}$. The different sensitivities of normal and transformed cells to TRAIL-induced apoptosis was proposed to be due to the expression of 'decoy' receptors in normal cells ${ }^{11}$. However, human hepatocytes express both DcR 1 and DcR 2, yet they are sensitive to TRAIL-induced apoptosis ${ }^{9}$.

The molecular mechanism underlying the TRAIL-induced signal-transduction pathway has not been well characterized $^{11}$. Studies in which DR4 or DR5 were overexpressed in human and mouse cells indicate that these receptors do not use FADD as an adaptor. However, a recent report $^{13}$ indicates that TRAIL induces the formation of a complex consisting of DR4, DR5, FADD and caspase 8, indicating that its apoptotic signal transduction pathway is similar to that used by Fas and TNFR1, at least in the cell lines examined. Whether or not TRAIL can activate NF- $\kappa \mathrm{B}$ is also controversial ${ }^{11}$, although it seems that TRAIL does not activate the immune system in vivo, at least in mouse and monkey. No knockout mice lacking TRAIL or its receptors have been reported, thus the physiological function of this death factor system remains elusive. The report by Jo et al..$^{9}$ indicates that administration of TRAIL could cause a severe, undesirable side effect-liver damage-in humans. It may not be too late to delay clinical trials until we have a better understanding of why some cells but not others are resistant to TRAIL.

1. Old, L.J. Tumor necrosis factor (TNF). Science 230, 630-632 (1985).

2. Vassalli, P. The pathophysiology of tumor necrosis factors. Annu. Rev. Immunol. 10, 411-452 (1992).

3. Trauth, B.C. et al. Monoclonal antibody-mediated tumor regression by induction of apoptosis. Science 245, 301-305 (1989).

4. Ogasawara, 1. et al. Lethal effect of the anti-Fas antibody in mice. Nature 364, 806-809 (1993).

5. Willey, S.R. et al. Identification and characterization of a new member of the TNF family that in-

duces apoptosis. Immunity 3, 673-682 (1995).

6. Pitti, R.M. et al. Induction of apoptosis by Apo-2 ligand, a new member of the tumor necrosis factor cytokine family. J. Biol. Chem. 271, 12687-12690 (1996).

7. Walczak, H. et al. Tumoricidal activity of tumor necrosis factor-related apoptosis-inducing ligand in vivo. Nature Med. 5, 157-163 (1999)

8. Ashkenazi, A. et al. Safety and antitumor activity of recombinant soluble Apo2 ligand. J. Clin. Invest. 104, 155-162 (1999).

9. Jo, M. et al. TNF-related apoptosis inducing ligand (TRAIL)-induced apoptosis in normal human hepatocytes. Nature Med. 6, 564-567 (2000)

10. Nagata, S. Apoptosis by death factor. Cell 88, 355-365 (1997)

11. Ashkenazi, A. \& Dixit, V.M. Death receptors: signaling and modulation. Science 281, 1305-1308 (1998).

12. Nagata, S. Fas ligand-induced apoptosis. Annu. Rev. Genetics 33, 29-55 (1999)

13. Bodmer, J.-L. et al. TRAIL receptor-2 signals apoptosis through FADD and caspase-8. Nature Cell Biol. 2, 241-243 (2000).

\section{Department of Genetics}

Osaka University Medical School

and Core Research for

Evolutional Science and Technology

Japan Science and Technology Corporation

2-2 Yamada-oka, Suita, Osaka 565-0871

Japan

Email:nagata@genetic.med.osaka-u.ac.jp

\section{Breaking point}

Ku80 is an essential participant, along with Ku70 and DNA-PKcs, in the non-homologous end-joining pathway, one mechanism that mammalian cells use to repair chromosome double-strand breaks (DSBs). Pro-T and pro-B lymphocytes from Ku80-/- mice are very susceptible to apoptosis and proliferate poorly, presumably because of an inability to repair DSBs. In the 31 March issue of Nature, Difilippantonio et al. further investigated Ku80 function by crossing Ku80-/- mice with mice deficient in p53, a protein involved in triggering cell cycle arrest and apoptosis in cells with unrepaired chromosome lesions. Loss of p53

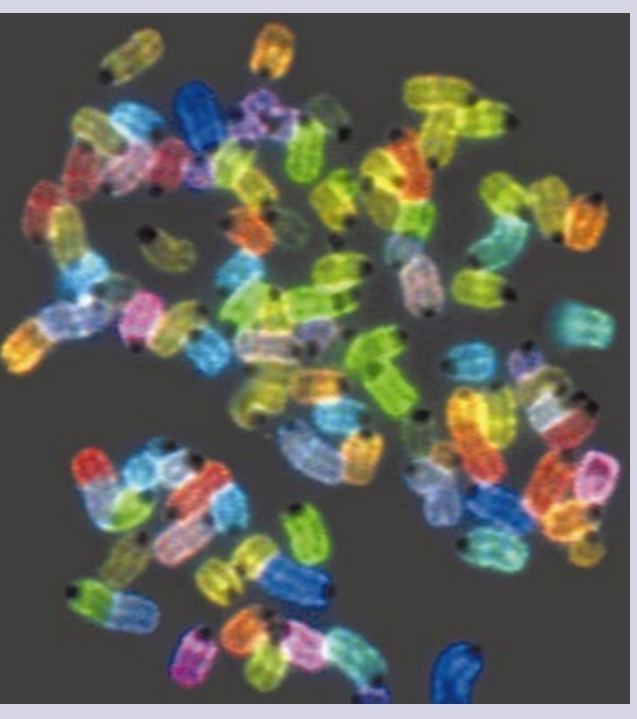
rescued the proliferation defects of Ku80-/- pro-B cells, but not pro-T cells, indicating that p53 is required for the induction of apoptosis in B-cell but not T-cell precursors with chromosome abnormalities induced by loss of Ku80. However, by 12 weeks of age, Ku80-/-p53-/- mice died from disseminated B-cell lymphoma. Chromosome analysis of Ku80-/- cells by spectral karyotyping (picture) showed a specific set of chromosomal translocations and amplifications involving, among others, $c-M y c$ and the $\lg \mathrm{H}$ locus, translocations that occur in Burkitt's lymphoma. Many other rearrangments of chromosomes, and even aneuploidy, were also seen in these cells. These findings place Ku80 in the company of ATM, BRCA1 and other proteins as genomic 'caretakers'.

Michael Ronemus 